\title{
Theoretical research and experimental verification on vibration and acoustic similarity of stiffened cylindrical shells
}

\author{
Bin Fang ${ }^{1,}$ a Zhemin $\mathrm{Jin}^{2, \mathrm{~b}}$ and Hanqin $\mathrm{Li}^{3, \mathrm{c}}$ \\ ${ }^{1,2,3}$ College of Warship and Ocean, Naval University of Engineering, No.717 Jiefang Road, Wuhan, \\ 430033, China \\ arobin_fb@163.com, b763487861@qq.com, ${ }^{c 58479221 @ q q . c o m ~}$
}

\begin{abstract}
Keywords: vibration, acoustics, similarity, siffened cylindrical shells.
Abstract. The employment of similitude theory to establish similarity among structural-acoustic systems can save considerable expense and time, if the proper scaling laws are found and validated. In this study necessary similarity conditions or scaling laws for dynamic analysis of stiffened cylindrical shell are developed through non-dimensionalizing the major variables in governing equations by Buckingham pi-theorem. In order to verify the similarity theory, two stiffened cylindrical shells, which are the model and prototype, are fabricated, and their frequency response functions (FRFs) of acceleration and sound pressure subjected to a random excitation are measured in a semi-anechoic chamber. Results show that FRFs of the model and prototype closely resemble each other compared throughout the frequency range and the scaling laws provide precise relationship between a full-scale prototype and its small-scale equivalent model.
\end{abstract}

\section{Introduction}

Dynamic response characteristics of structural-acoustic systems are one of the fundamental requirements in designing and evaluating noise control measures. Finding dynamic response, including frequency response functions (FRFs) of acceleration and sound pressure, through performing acoustic test in case of heavy and huge systems, called the prototype, is very difficult to do and also requires advanced and huge test instrumentations and considerable expense and time. The employment of similitude theory to establish similarity among structural-acoustic systems can save considerable expense and time, if the proper scaling laws are found and validated. Similarity conditions or scaling laws provide the relationship between the prototype and its scale models. This relationship must be based on the existence of certain parameters that control the behavior of the prototype. If such parameters exist, a scaled down model can be built, which will duplicate the response of the full scale prototype. The two systems are then said to be similar. The theory of similitude includes a consideration of the conditions under which the behavior of two separate systems will be similar. Considering the significant role of stiffened cylindrical shell in various types of structural-acoustic systems, practical application of scaled down stiffened cylindrical shells and the importance of establishing a similarity and finding proper scaling laws will be evident. Due to the large number of design parameters in stiffened cylindrical shells, the identification of the principal scaling laws only through dimensional analysis is difficult. Similitude theory based on the governing equation of structural-acoustic systems is more direct and simpler in execution, so both the dimensional analysis and the equations analysis will be used in this paper.

Studies particularly concerning the use of scaled down shell structures have been conducted in the past. Soedel investigated similitude requirements for vibrating thin shells [1]. Prediction of shell vibration response in cross-ply cylindrical shells using scaled down models, based on partial similitude was accomplished by Rezaeepazhand [2]. In a similar investigation presented by the same authors, vibration response of laminated cylindrical shells with double curvature was investigated based on structural similitude [3]. Effect of extensive use of welding on buckling behavior of large cylindrical shells constructed from a large number of curved panels was investigated using scale models by Teng [4]. Wang used dimensional theory to obtain the similarity conditions between model and prototype for an underwater stiffened cylinder [5]. Jha developed the necessary similarity 
conditions for dynamic testing of scaled structures [6]. Torkamani used the Donnell-type nonlinear strain-displacement relations along with the smearing theory to develop the similitude theory for free vibrations of orthogonally stiffened cylindrical shells [7]. And in order to overcome the difficulties encountered in fabricating small-scaled stiffeners, design of equivalent cross section was explored that in spite of having much simpler profile, offers the same vibration effect in free vibration of stiffened shells. A scaling approach to predict high-frequency mean responses of vibration systems was analyzed by Li [8]. Many research activities had been conducted on scale-down modeling of dynamic and static behavior of other structural systems. Rosa proposed a similitude for analysis of the dynamic response of acousto-elastic assemblies, invoking the energy distribution approach which allows the exact representation of all the fundamental parameters in terms of modal coordinates [9]. The scaling laws for physical modeling of anti-symmetric cross-ply laminated circular cylindrical shells for buckling and free vibration were developed by Variddhi [10].

In the present study, scaling laws have been derived for the dynamic analysis of structural-acoustic system and different types of possible relaxations are taken into consideration to meet the scaling conflicts encountered for dynamic analysis. For the purpose of experimental validation of the methodology, stiffened cylindrical shells of the model and prototype are fabricated, and their FRFs of acceleration and sound pressure subjected to a random excitation are measured in a semi-anechoic chamber. Results show that the FRFs of the model and the prototype closely resemble each other compared throughout the frequency range.

\section{Scaling laws of stiffened cylindrical shells}

Scaling laws can be confined to the following steps: list all relevant variables involved in the physical problem, establish by dimensional analysis all pi-terms, list the given values and (or) ranges of prototype variables and establish the scaling factors for all variables.

The governing equation for acoustic radiation of structure can be expressed as Eq. 1:

$$
g\left(\pi_{1}, \pi_{2}, \mathrm{~L}, \pi_{n-m}\right)=0 .
$$

where $\pi_{1}, \pi_{2}, \mathrm{~L}, \pi_{n-m}$ are dimensionless products of the $n$ physical variables, and $m$ is the number of fundamental dimensions that are involved in the physical problem. In general, three independent scale factors, which represent three fundamental dimensions, namely mass $(M)$, length $(L)$ and time $(T)$, need to be selected for designing the scaled model. The dimensions of other quantities can be expressed in terms these three fundamental dimensions.

According to similitude requirement, the pi-terms $\left(\pi_{1}, \pi_{2}, \mathrm{~L}, \pi_{n-m}\right)$ must be equal in the model and the prototype, in order to match the functional relationship between them. It is not always clear which variables should be included in the pi-terms, so the governing equations for vibration and acoustic radiation are introduced. For the forced vibration of damped multiple-degrees-of-freedoms systems, governing equation can be expressed as Eq. 2 [11]:

$$
[M]\{+[C]\{l \&+[K]\{u\}=\{F\} .
$$

where $M$ is the mass matrix of the structure, $C$ is the damping coefficient matrix, $K$ is the stiffness matrix, $\{u\}$ is the column matrix for displacement, $\{u\}$ is the first-order derivative for displacement, $\{$ is the second-order derivative for displacement, $\{F\}$ is the column matrix of applied force including the excitation force. There are many variables in the equation including the density of material $\rho$, the geometry dimension of structure $l$, the Young's modulus $E$ and Poisson's ratiov .

The governing equation for acoustic radiation of structure can be expressed as Eq. 3 [12]:

$$
\nabla^{2} p-\frac{1}{c_{0}^{2}} \frac{D^{2}}{D t^{2}} p=\nabla \cdot \boldsymbol{F}-\rho_{0} \frac{D q}{D t} .
$$


where $V$ is mean velocity of the fluid medium through which the sound propagates, $p$ is its pressure, $\rho_{0}$ is its density, $c_{0}$ is the speed of sound, $\boldsymbol{F}$ denotes an externally applied volume force, $x$ is the direction of flow.

If the material behavior is assumed to be linearly elastic, the acceleration of stiffened cylindrical shells can be expressed as Eq. 4:

$$
A=f_{a}\left(D, C, F_{0}, r, l, \rho, \omega\right) .
$$

If the mean inflow is not considered, the radiation sound pressure can be expressed as Eq. 5:

$$
p=f_{p}\left(D, C, F_{0}, r, \rho_{0}, c_{0}, Z, l, \omega, \rho\right) .
$$

The nine required pi-terms based on Eq. 4 and Eq. 5 are given by

$$
\begin{aligned}
& \pi_{1}=\frac{A}{\omega^{2} l}, \pi_{2}=\frac{p}{\rho \omega^{2} l^{2}}, \pi_{3}=\frac{D}{\rho \omega^{2} l^{5}}, \pi_{4}=\frac{C}{\rho \omega l^{3}}, \pi_{5}=\frac{F_{0}}{\rho \omega^{2} l^{4}} . \\
& \pi_{6}=\frac{r}{l}, \pi_{7}=\frac{\rho_{0}}{\rho}, \pi_{8}=\frac{c_{0}}{\omega l}, \pi_{9}=\frac{Z}{l}
\end{aligned}
$$

So the Eq. 5 can be written in form of the dimensionless pi-term. Scaling laws now can be readily developed by applying similarity conditions on these pi-terms. By introducing scale factor, $\lambda_{i}$ as the ratio of a particular variable $x_{i m}$ in model to the corresponding variable $x_{i p}$ in prototype, the variables of the model can be written as $x_{i p}=\lambda_{i} x_{i m}$, where subscripts $m$ and $p$ refer to model and prototype, respectively. The necessary scaling laws for vibration and acoustic radiation of stiffened cylindrical shells may be obtained as Eq. 7:

$$
\begin{aligned}
& D_{\psi}=\frac{\left(\pi_{3}\right)_{p}}{\left(\pi_{3}\right)_{m}}=1, C_{\psi}=\frac{\left(\pi_{4}\right)_{p}}{\left(\pi_{4}\right)_{m}}=1, F_{0 \psi}=\frac{\left(\pi_{5}\right)_{p}}{\left(\pi_{5}\right)_{m}}=1, r_{\psi}=\frac{\left(\pi_{6}\right)_{p}}{\left(\pi_{6}\right)_{m}}=1 . \\
& \rho_{\psi}=\frac{\left(\pi_{7}\right)_{p}}{\left(\pi_{7}\right)_{m}}=1, c_{0 \psi}=\frac{\left(\pi_{8}\right)_{p}}{\left(\pi_{8}\right)_{m}}=1, Z_{\psi}=\frac{\left(\pi_{9}\right)_{p}}{\left(\pi_{9}\right)_{m}}=1
\end{aligned}
$$

Eq. 7 is the necessary and sufficient conditions for complete similarity between model and prototype. When at least one of the similarity conditions cannot be satisfied, partial similarity is achieved and the model which has some relaxation in similarity conditions is called a distorted model.

\section{Application of the scaling laws for stiffened cylindrical shell}

Stiffened cylindrical shell must be designed, loaded and interpreted according to the scaling laws that relate the model to the prototype. Replica model is a physical model of the prototype which is geometrically similar in all aspects to the prototype and employs identically the same materials at similar locations. Taking the geometrical scale factor equal to $k$, and by assuming that the BCs (boundary conditions) and loading conditions are similar for model and prototype, inputs to the similarity problem can be extracted as follows.

Geometric similarity. The scale factors of geometry are

$$
\lambda_{l}=\frac{R_{p}}{R_{m}}=\mathrm{L}=k, \lambda_{r}=\frac{r_{p}}{r_{m}}=k .
$$

It means that the model is geometrically similar in all aspects to the prototype and the location of sensors, such as accelerometers and microphones, is corresponding to those of the prototype.

Kinematic similarity. Condition where the velocity ratio is a constant between all corresponding points can be extracted as follows for this problem. 


$$
\lambda_{c_{0}}=\frac{c_{0 p}}{c_{0 m}}=1 \text {. }
$$

It means that the model and prototype will be in the same fluid medium, such as air or water.

Dynamic similarity. Forces which act on corresponding masses in the model and prototype are in the same ratio. Suppose that for this problem, exciting force is present, dynamic similarity requires that, at corresponding points on the structure.

$$
\lambda_{F_{0}}=\frac{F_{0 p}}{F_{0 m}}=k^{2} .
$$

BCs similarity. If the acoustic test is proceeding in a semi-anechoic chamber with a hard boundary or in water with free surface, the BCs similarity requires that

$$
\lambda_{Z}=\frac{Z_{p}}{Z_{m}}=k
$$

Time similarity. Condition where the time ratio is a constant between the model and prototype can be extracted as follows in form of frequency.

$$
\lambda_{\omega}=\frac{\omega_{p}}{\omega_{m}}=k^{-1} .
$$

It means that the frequency ratio of the model and prototype will be equal to the inverse of geometrical scale factor, i.e., $k^{-1}$.

Material similarity. Condition where the material ratio is a constant between the model and prototype can be extracted as follows in form of Young's modulus, Poison's ratio, stiffness, density and mass. Considering that the material of model and prototype are the same, so

$$
\lambda_{E}=\frac{E_{p}}{E_{m}}=1, \lambda_{\mathrm{v}}=\frac{v_{p}}{v_{m}}=1, \lambda_{\rho}=\frac{\rho_{p}}{\rho_{m}}=1, \lambda_{\rho_{0}}=\frac{\rho_{0 p}}{\rho_{0 m}}=1, \lambda_{M}=\frac{M_{p}}{M_{m}}=k^{3}, \lambda_{K}=\frac{K_{p}}{K_{m}}=k .
$$

The flexural stiffness of stiffened cylindrical shells can be expressed as

$$
D=\frac{E t_{e}^{3}}{12\left(1-v^{2}\right)} \text {. }
$$

where $t_{e}$ is the equivalent thickness of stiffened cylindrical shells. The stiffness scale factor is

$$
\lambda_{D}=\frac{D_{p}}{D_{m}}=k^{3}
$$

When the Eq. 8 15 are valid, scaling laws expressed below can be satisfied.

$$
\begin{aligned}
& D_{\psi}=\frac{\lambda_{D}}{\lambda_{\rho} \lambda_{\omega}{ }^{2} \lambda_{l}{ }^{5}}=1, F_{0 \psi}=\frac{\lambda_{F_{0}}}{\lambda_{\rho} \lambda_{\omega}{ }^{2} \lambda_{l}{ }^{4}}=1, r_{\psi}=\frac{\lambda_{r}}{\lambda_{l}}=1, \\
& \rho_{\psi}=\frac{\lambda_{\rho_{0}}}{\lambda_{\rho}}=1, c_{0 \psi}=\frac{\lambda_{c_{0}}}{\lambda_{\omega} \lambda_{l}}=1, Z_{\psi}=\frac{\lambda_{Z}}{\lambda_{l}}=1
\end{aligned}
$$

For the vibration systems without damping, it will be complete similarity between model and prototype, and the scale factor for acceleration and radiation sound pressure can be expressed as

$$
\lambda_{A}=\frac{A_{p}}{A_{m}}=k^{-1}, \lambda_{p}=\frac{p_{p}}{p_{m}}=1 .
$$


But it is a complicated problem for the vibration systems with damping, which requires

$$
C_{\psi}=\frac{\lambda_{C}}{\lambda_{\rho} \lambda_{\omega} \lambda_{l}^{3}}=\frac{\lambda_{C}}{1 k^{-1} k^{3}}=\frac{\lambda_{C}}{k^{2}}=1 \Rightarrow \lambda_{C}=k^{2} .
$$

The form of damping coefficient for vibration systems is not unique. If the Rayleigh's damping is introduced, the scale factor of damping coefficient will be [11]

$$
\lambda_{C}=\frac{C_{p}}{C_{m}}=\frac{(\alpha M+\beta K)_{p}}{(\alpha M+\beta K)_{m}}=\frac{\alpha \lambda_{M} M_{m}+\beta \lambda_{K} K_{m}}{\alpha M_{m}+\beta K_{m}}=\frac{\alpha k^{3} M_{m}+\beta k K_{m}}{\alpha M_{m}+\beta K_{m}} .
$$

Vibrations of the stiffened cylindrical shells with damping cannot be complete similarity between model and prototype, only if $k=1$. Fortunately, for metal materials, such as steel, which is used to fabricate the stiffened cylindrical shells, the damping fraction of critical damping $\zeta=c / c_{c}<1$, where $c_{c}=2 \sqrt{\mathrm{km}}=2 m \omega_{n}, \omega_{n}$ is the angular natural frequency of vibration system [11]. The damped natural frequency related to the undamped natural frequency can be derived below

$$
\omega_{d}=\omega_{n}\left(1-\zeta^{2}\right)^{1 / 2} \Rightarrow \omega_{d} \approx \omega_{n} \text {, if } \zeta<1
$$

The natural frequency of vibration with damping will be equal to that of vibration without damping. So the scale factor of damped natural frequency is

$$
\lambda_{\omega_{d}}=\frac{\omega_{d p}}{\omega_{d m}} \approx k^{-1} .
$$

For maintaining complete similarity becomes cumbersome and impractical, it was suggested that neglecting the effects of damping in the stiffened cylindrical shells provides reasonably good results if the frequency characteristics are considered particularly.

In the case of similarity conditions being satisfied, the non-dimensional frequency parameter defined as below [7]

$$
\Omega=\omega \sqrt{\frac{\bar{M} l_{c}^{4}}{D}} .
$$

$\Omega$ have the same value in model and prototype, where $\bar{M}$ is the averaged mass per unit area of the stiffened cylindrical shell, $l_{c}$ is the length of cylinder.

The non-dimensional acceleration $\Lambda$ and non-dimensional radiation sound pressure $\Theta$ defined as below

$$
\Lambda=A M / F_{0}, \Theta=p L_{d}^{2} / F_{0} \text {. }
$$

$\Lambda$ and $\Theta$ have the same value in model and prototype when the complete similarity is achieved, where $L_{d}$ is the distance between microphones and the center of cylinder and $M$ is the mass of stiffened cylindrical shell.

\section{Experimental validation of the scaling laws}

For the purpose of experimental validation of the methodology as applied here, two cylindrical shells are fabricated. The first shell denoted as prototype is a stiffened cylindrical shell made of steel, having nine equally spaced internal rectangle shape ribs (Fig.1). The second shell denoted as model is a $1 / 2$-scale model of the prototype, made of steel, which is the scale down of the prototype on geometry. 

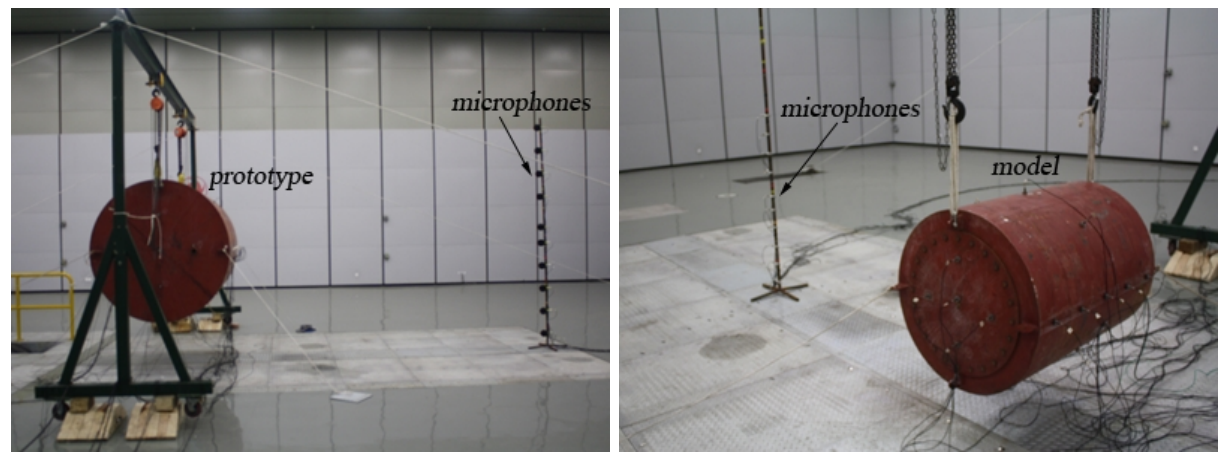

Fig. 1 Prototype and model in a semi-anechoic chamber

The excitation force is measured by a force transducer. The acceleration response is measured using piezoelectric accelerometers in the longitudinal and radial directions on 38 points of the prototype and the corresponding points of the model. The sound pressure is measured by microphones on lateral directions on 10 points of the prototype and the corresponding points of the model. All the signals are collected by the signal collection devices and analyzer software is used to extract FRFs. The vibration response and acoustic radiation of the model and the prototype can be compared throughout the frequency range of excitation. A number of such plots, comparing the FRFs of some distinct points on the prototype and their corresponding points on the model, measured during the test are presented in Fig. 2 and Fig. 3.
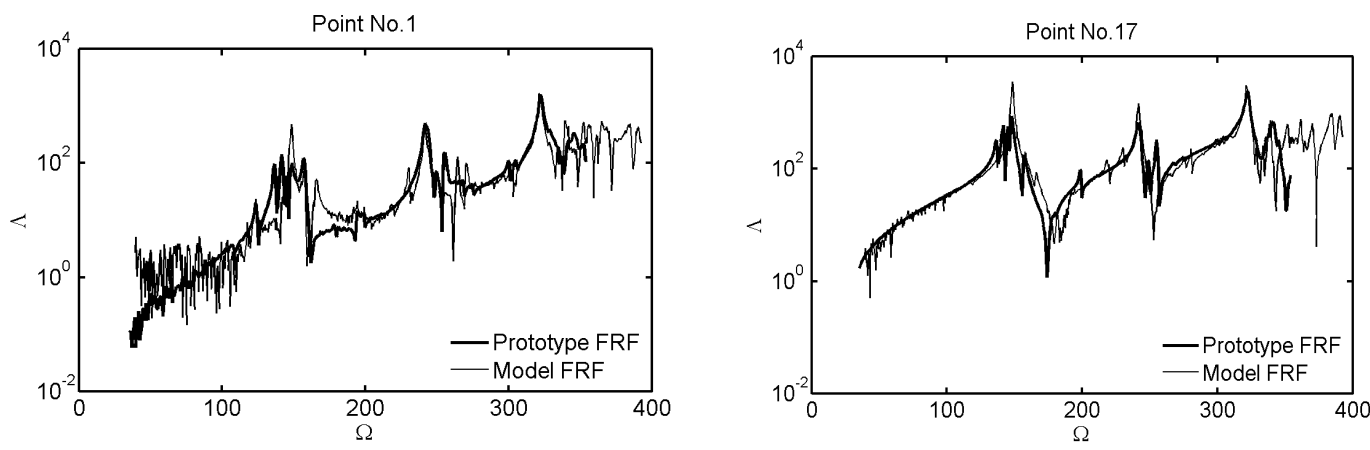

Fig. 2 Acceleration FRFs of prototype and model.
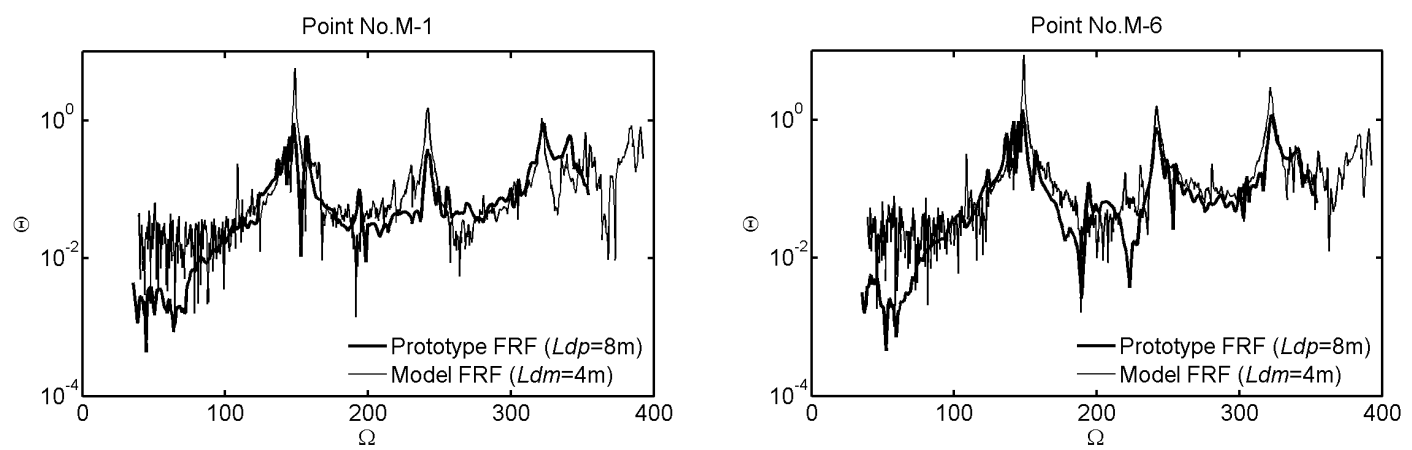

Fig. 3 Sound pressure FRFs of prototype and model.

The behavior of the model and prototype closely resemble each other, but in some cases such as the response amplitude peaks there exists inconsistency. In this case, since the less-than-perfect value is occurred for the response amplitude peaks, the reason probably is the dissimilar of damping coefficient between the model and the prototype.

\section{Conclusions}

In this study, necessary similarity conditions or scaling laws for dynamic analysis of stiffened cylindrical shell are developed through non-dimensionalizing the major variables in governing equations by Buckingham pi-theorem. Based on the similarity conditions or scaling laws, the relation between the acceleration and the sound pressure of a scale model and those of its prototype are 
obtained. For maintaining complete similarity becomes cumbersome and impractical, it was suggested analytically and experimentally that neglecting the effects of damping in the stiffened cylindrical shells provides reasonably good results if the frequency characteristics are considered particularly. For the purpose of experimental validation of the methodology, stiffened cylindrical shells of the model and prototype are fabricated, and their FRFs of acceleration and sound pressure subjected to a random excitation are measured in a semi-anechoic chamber. Results show that the FRFs of the model and prototype closely resemble each other compared throughout the frequency range. It means that the scaling laws provide precise relationship between a full-scale prototype and its small-scale equivalent model for the frequency characteristics of dynamic response, and can be used to extrapolate the experimental data of a small inexpensive and testable model into design information for a large prototype.

\section{Acknowledgment}

Authors would like to gratefully acknowledge Mr. Bo Wang, Mr. Qiongfeng Xu, Mr. Chao Li and $\mathrm{Mr}$. Lu Tan for their effort in doing experiments.

\section{References}

[1] W. Soedel: Similitude Approximations for Vibrating Thin Shells. J. Acoust. Soc. Am. Vol. 49(1971), p.1535-1541.

[2] J. Rezaeepazhand, G.J. Simitses, J.H. Starnes: Design of scaled down models for predicting shell vibrations response. J. Sound Vib. Vol. 195 (1996), p. 301-311.

[3] J. Rezaeepazhand, G.J. Simitses: Structural similitude for vibration response of laminated cylindrical shells with double curvature. Composites Part B Vol. 28(1997), p. 195-200.

[4] J. G. Teng, X Lin: Fabrication of small models of large cylinders with extensive welding for buckling experiments. Thin Wall Struct. Vol. 43(2005), p. 1091-114.

[5] S. Wang: Study on the acoustic similitude of the underwater complex shell structure. J. Acoust. Soc. Am. Vol. 119(2006), p. 3419-3419.

[6] A. Jha, R. Sedaghati, R. Bhat: Analysis and dynamic testing of structures subjected to vibration and shock using scale models. Canadian Aeronautics and Space Journal. Vol. 52(2006), p. 95-108.

[7] Sh. Torkamani, H.M. Navazi, A.A. Fafari, M. Bagheri: Structural similitude in free vibration of orthogonally stiffened cylindrical shells. Thin Wall Struct. Vol. 47(2009), p. 1316-1330.

[8] Xianhui Li: A scaling approach for the prediction of high-frequency mean responses of vibration systems. J. Acoust. Soc. Am. Vol. 127(2010), p. 209- 214.

[9] S.D. Rosa, F. Franco, X. Li, T. Polito: A similitude for structural acoustic enclosures. Mech. Syst. Signal Pr. Vol. 30 (2012), p. 330-342.

[10]U. Variddhi, S. Pairod: Similitude invariants and scaling laws for buckling experiments on anti-symmetrically laminated plated subjected to biaxial loading. Compos. Struct. Vol. 59(2003), p. $455-465$.

[11]G. P. Allan, L. T. Paez, in: Harris' Shock and Vibration Handbook, 6th.ed. edtied by McGraw-HILL International Book Company, New York (2009), in press.

[12] M. E. Goldstein, in: Aeroacoustics. edtied by McGraw-HILL International Book Company, New York (1976), in press. 